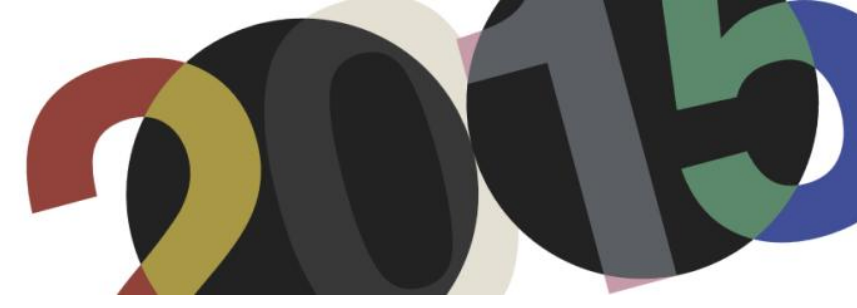

DOI: http://dx.doi.org/10.4995/LC2015.2015.628

\title{
La casa y el recinto. Un proceso abierto a través del proyecto para la Residencia Peyrissac en una explotación agrícola en Argelia
}

\author{
C. Martínez Arroyo*, R. Pemjean Muñoz*, J.P. Sanz Alarcón** \\ * Escuela Técnica Superior de Arquitectura de Madrid \\ ** Escuela Técnica Superior de Arquitectura y Edificación de Cartagena
}

\begin{abstract}
Resumen: El objeto de estudio de esta investigación es el proyecto residencial no construido que realiza Le Corbusier en 1942 para una explotación agrícola cerca de Cherchell, Argelia. A partir del conjunto de dibujos que constituye la documentación gráfica existente sobre esta propuesta, el estudio aquí desarrollado basa su interés en describir el proyecto como proceso abierto de reflexión arquitectónica sobre la idea de la casa dentro de un recinto acotado. Se realiza un análisis pormenorizado de los distintos mecanismos y temas de proyecto empleados por el arquitecto mediante el desarrollo de nuevos planos interpretativos de la documentación original. De este modo se encuentran tanto los invariantes como las distintas versiones que construyen el desarrollo de la propuesta. Finalmente se concluye mediante una síntesis crítica en la que se relacionan los ámbitos arquitectónicos estudiados con soluciones análogas dentro tanto dentro del conjunto de la obra de Le Corbusier como con ejemplos paradigmáticos del panorama arquitectónico.
\end{abstract}

Abstract: The object of study of this research is the not built residential project designed by Le Corbusier in 1942 for a farm near Cherchell, Algeria. From the drawings that constitute the existing graphic documentation of this proposal, this study based its interest in describing the project as an open process of architectural reflection on the idea of the house within a bounded enclosure. A detailed analysis of the different mechanisms and project themes used by the architect through the developing of new interpretive drawings of the original documentation. In this way the invariants and different versions that built the development of the proposal are present. Finally we conclude with a critical synthesis where we relate the architectural areas studied with similar solutions from Le Corbusier works and paradigmatic examples of the architectural scene.

Palabras clave: Le Corbusier; Argelia; Peyrissac; casa; recinto; patio. Keywords: Le Corbusier; Argelia; Peyrissac; house; enclosure; court.

\section{Introducción}

El objeto de estudio de esta investigación es el proyecto residencial no construido que realiza Le Corbusier en 1942 para una explotación agrícola cerca de Cherchell, Argelia. Se trata de un proyecto basado en la relectura de la arquitectura tradicional mediterránea desde la óptica y el léxico moderno ${ }^{1}$. La propuesta reflexiona sobre cómo realizar un alojamiento adecuado al clima, en función del sol y los vientos, las condiciones y medios locales. El ejercicio práctico desarrollado por Le Corbusier plantea un hábitat defensivo e interior en relación con la soledad del lugar junto a una arquitectura abierta y vinculada con la amplitud del horizonte que le rodea. A

\footnotetext{
${ }^{1}$ Junto a los croquis del proyecto, se trascriben las palabras de Le Corbusier: En la construcción moderna, encontramos la relación entre el paisaje, el clima y la tradición. Véase Le Corbusier: Oeuvre complète Volume 4 1938-46, 11th edition. Basel: Birkhäuser-Publishers for Architecture, 1999, p. 123.
} 
partir del conjunto de dibujos que constituye la documentación gráfica existente sobre esta propuesta, el estudio aquí desarrollado se basa en el interés del desarrollo del proyecto como proceso abierto de reflexión.

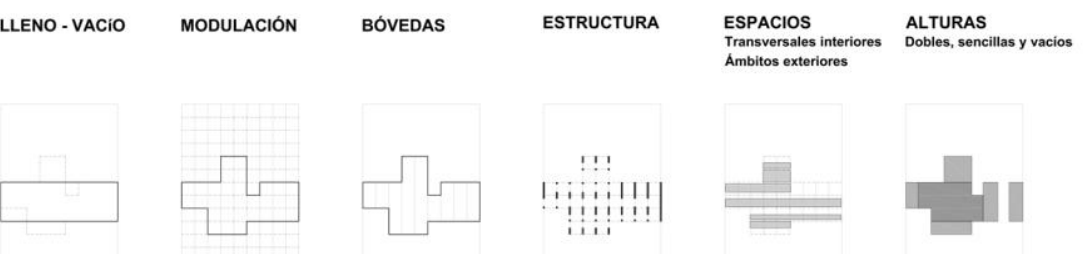

CASA
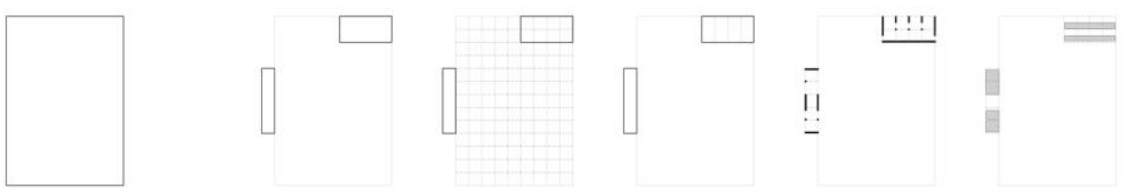

RECINTO

MIRADORES
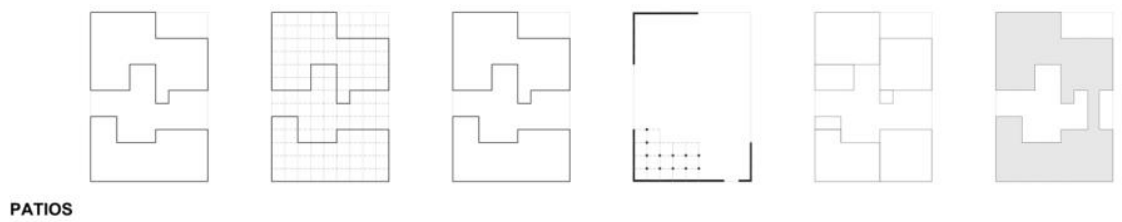

1. Tabla comparativa de los distintos mecanismos arquitectónicos empleados en el proyecto.

Este procedimiento se configura mediante la continua transformación y definición de diversos mecanismos de proyecto a través del dibujo como medio de pensamiento. Estos temas de proyecto que aparecen en los croquis suponen un invariante a lo largo de todo el proceso, adquiriendo distintas versiones que dan lugar a un proyecto abierto e inconcluso.

El objetivo del trabajo es definir el grado de variación e intensidad con el que aparecen los mecanismos utilizados por Le Corbusier y de este modo demostrar mediante el análisis del proyecto los parámetros arquitectónicos empleados. Esta hipótesis de trabajo se traduce, y por tanto aparece reflejada, en la estructura de la investigación. De este modo, la comunicación queda organizada en dos partes diferenciadas.

Una primera de carácter analítico organizada sobre los cuatro elementos fundamentales que estructuran y configuran el proyecto: el recinto, la casa, los miradores y los patios. Esta parte del trabajo se desarrolla a partir de la documentación original existente (28 croquis en planta, sección, axonometría o perspectiva) ${ }^{2}$. Estos planos construyen el guión en torno a la idea de casa dispuesta en el interior de un recinto acotado. Se estudia en profundidad el contenido de cada uno de ellos, extrayendo los datos que ponen de manifiesto los sistemas y mecanismos espaciales, constructivos y materiales que organizan la propuesta. Para ello se realiza una reconstrucción del proyecto mediante la elaboración de una nueva planimetría analítica.

\footnotetext{
${ }^{2}$ Cabe destacar la importancia que adquiere la publicación de este proyecto en la obra completa de Le Corbusier ya que se presenta a lo largo de 8 páginas mediante una selección de dibujos. En este estudio se ha tenido en cuenta el contenido completo de los planos y dibujos originales del proyecto, lo cual ha posibilitado acceder a distintas versiones anteriores respecto a las conocidas por las publicaciones.
} 

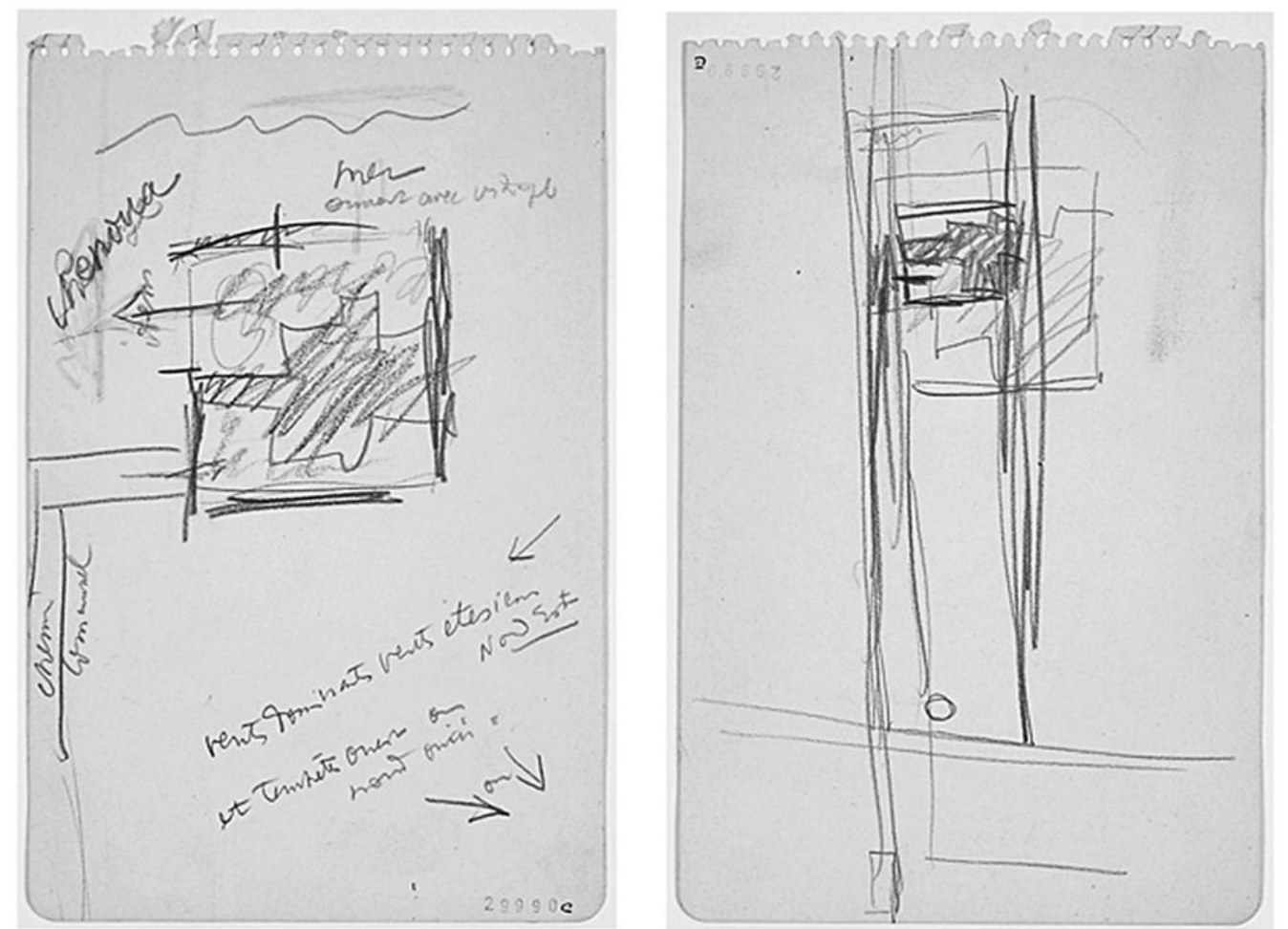

2. Dibujos originales FLC 29990C-CFLC-ADAGP y FLC 29990E-CFLC-ADAGP que muestran la relación del recinto con el paisaje próximo.

La segunda parte, a modo de síntesis crítica, se estructura sobre los diferentes temas de proyecto encontrados y contextualizados dentro de la obra de Le Corbusier: la configuración de transiciones espaciales desde el exterior hasta el interior a través de espacios intermedios haciendo de la casa un umbral, el recinto como muro grueso convertido en espacio destinado a la mirada extrovertida en relación con el horizonte, la ruptura del espacio lineal y unitario bajo las bóvedas de cañón consiguiendo visiones diagonales en los ámbitos de la vivienda; o la combinación gradual de llenos y vacíos bajo la idea de patio y pabellón.

\section{Análisis}

Tal y como se ha señalado anteriormente, los cuatros ámbitos de reflexión permanente y fundamentales que encontramos en los dibujos de Le Corbusier en el proyecto, y que son objeto de análisis pormenorizado en esta investigación, son: la materialización del recinto a través de una tapia recortada, la definición de una masa edificada que forma la casa o espacios interiores, la especialización de los vacíos a modo de patios que se delimitan dentro del recinto y la construcción de espacios de control sobre la mirada lejana hacia el horizonte.

\subsection{El recinto}

La primera idea que se incorpora al desarrollo del proyecto y que nunca desaparece es la de crear un recinto cerrado y materializar un habitar introvertido en el interior entre jardines. En los dibujos se hace incidencia sobre la configuración del recinto como lugar acotado y finito ${ }^{3}$. La casa se propone en apariencia defensiva y cerrada pero a la vez abierta mediante, tanto con un torreón para vigilar a los merodeadores como con miradores hacia el paisaje. La tapia aparece como elemento terso e infranqueable en primer plano mientras que las bóvedas de la

\footnotetext{
${ }^{3}$ Plano original FLC 29990C.
} 
cubierta de los elementos miradores aparecen como elementos que flotan sobre la tapia en un segundo estrato de altura $^{4}$. Esta imagen de prisma regular con elementos curvos configurando la cubierta resulta análoga a la de la villa Savoye.

Los croquis hacen hincapié en el valor del emplazamiento en relación con la inclusión del recinto habitado en el paisaje dando lugar a un diálogo explícito entre lo natural y lo artificial. Así, por ejemplo, cuando el recinto de la casa aparece dibujado de forma prolongada hacia el paisaje circundante por la longitud de los muros que lo delimitan, se estable una clara ligazón entre la pieza arquitectónica y su lugar de inserción ${ }^{5}$.

La construcción de un límite dentro del cual se inserta la casa hace que en el proyecto se maneje constantemente la dialéctica entre la escala doméstica interior y la gran escala exterior en relación con el medio natural. Esta dualidad se ve representada por aquellos dibujos que muestran la imagen exterior de la casa frente a los croquis que inciden en la visión desde el interior de la casa hacia el paisaje ${ }^{6}$. Por tanto el recinto adquiere una doble versión contrapuesta: la de ocultar el interior desde el exterior; y la de abrir los espacios de la casa hacia las vistas.
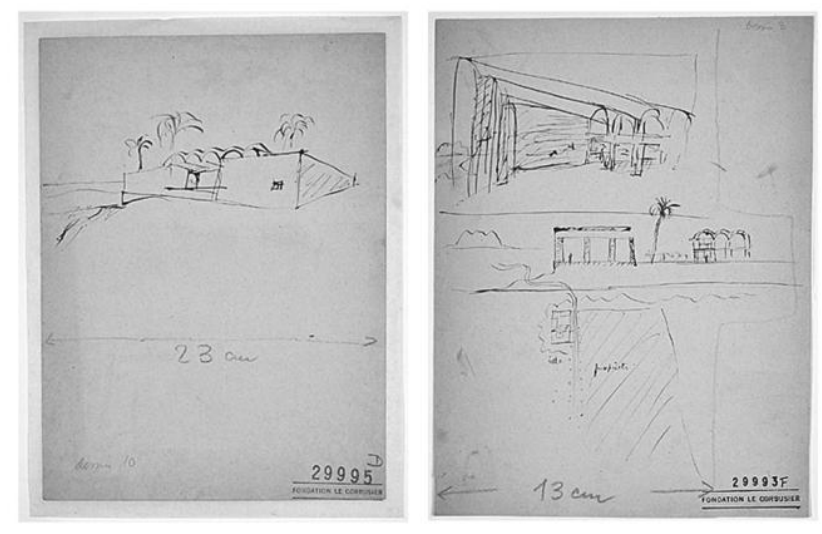

\section{Dibujos originales FLC 29995D-OFLC-ADAGP y FLC 29993F-OFLC-ADAGP.}

La configuración geométrica del recinto se realiza por la determinación de un límite o perímetro construido a partir de una matriz modulada. Dentro de esta trama regular de 4 metros de lado se van definiendo las distintas versiones que adopta. El recinto aparece siempre como una forma rectangular ortodoxa sobre la que se delimitan zonas ciegas y otras permeables. A lo largo del proceso se muestra como el recinto se va reconfigurando, abriendo y cerrando, en función de las relaciones con el exterior ${ }^{7}$.

\footnotetext{
${ }^{4}$ Plano original FLC 29993H.

${ }^{5}$ Plano original FLC 29990E.

${ }^{6}$ Como por ejemplo sucede en las perspectivas exteriores del plano original FLC 29995D frente a la visión desde el interior de la casa que se dibuja en el FLC 29993F.

${ }^{7}$ En el exterior del recinto queda una gran plantación de naranjos y de tomates sobre una plataforma limitada por un acantilado sobre el borde del mar. También aparece una piscina que hará las veces de aljibe de agua para regar la vegetación circundante. La tapia del recinto se desmaterializa para conectar el interior de los patios con estos elementos del exterior.
} 


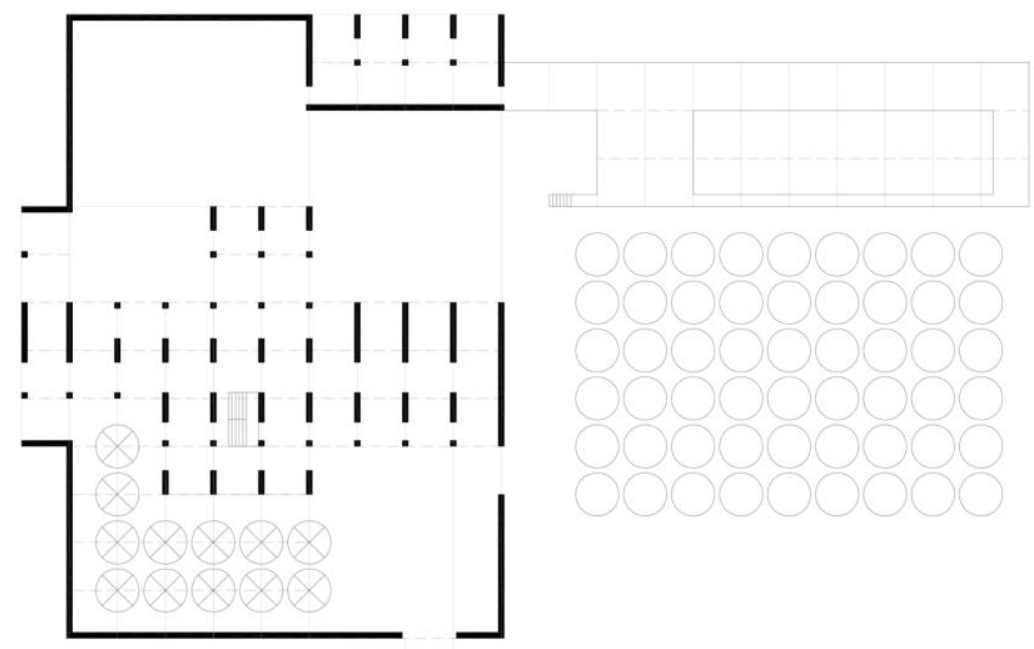

4. Planta baja de la vivienda y los patios realizada a partir de la documentación original sobre la propuesta más desarrollada.

Una vez construida la idea de recinto, otra de las preocupaciones asociadas al mismo es la forma de acceso. El camino de entrada aparece de forma recurrente en los dibujos quedando siempre latente la reflexión sobre este aspecto. Existen dos principales versiones sobre la manera de acceder al recinto. Por un lado aquellos dibujos que muestran una disposición lateral del recorte de entrada en la tapia ${ }^{8}$; y por otra parte, como sucede en la versión más desarrollada, la determinación de un acceso frontal a la dirección de llegada al recinto por el camino de aproximación. La indecisión sobre este aspecto se ve reflejada permanentemente en toda la documentación gráfica existente del proyecto. Ambas formas de acceder influyen directamente en la configuración de los espacios vacíos interiores al recinto que aparecen inmediatamente a continuación de la entrada a través de la tapia. En los accesos laterales se produce el desarrollo de la circulación tangente a la tapia por el exterior creándose un recinto o explanada de acceso previo a modo de vestíbulo. Sin embargo, en las versiones en las que se produce el acceso frontal se crea un patio de entrada pero esta vez en el interior del recinto. Esto hace que se fragmente y se especialice el uso o programa de los patios interiores. Así, estos ámbitos rompen su unidad y se separan según su utilización. La circulación en este caso queda siempre en el interior de la tapia, en un caso tangente a ella y en otro, en la propuesta más desarrollada, separada del borde creándose una perspectiva lineal a través de una secuencia conformada por un doble patio de acceso, uno previo de coches y otro posterior que da servicio a los ámbitos de la vivienda ${ }^{9}$.

\subsection{La casa}

La casa se configura como la suma de módulos construidos dentro de la trama regular. Su forma queda determinada por la relación intrínseca entre los llenos y vacíos mediante mecanismo de adición de piezas. Dentro

\footnotetext{
${ }^{8}$ Tal y como se refleja en el croquis FLC 29992D.

${ }^{9}$ En la planta baja del plano FLC 29995A se puede apreciar la disposición de dichos patios.
} 
del proceso de trabajo se muestra como un sistema dinámico, reconfigurable y no definitivo. La posición de la casa dentro del recinto si que aparece como un invariante en todos los dibujos del proceso del proyecto, quedando establecida en un punto intermedio del mismo, determinando así en todos los casos un vacío delantero frente a uno trasero. La casa queda definida entre dos patios. El lleno divide y fragmenta en dos partes al vacío ${ }^{10}$.

La estructura que materializa los distintos espacios de la casa queda asociada a la idea de matriz modular. De este modo aparece un orden longitudinal del espacio asociado a un tipo de sección estructural en forma de bóveda de cañón ${ }^{11}$. Uno de los proceso más interesantes del proyecto es la permanente indefinición sobre la direccionalidad de las bóvedas, ya que dependiendo del dibujo que analicemos, éstas aparecen según el eje esteoeste, y en otras ocasiones colocadas norte-sur ${ }^{12}$. Esta doble direccionalidad permite reconocer dos tipos de proyectos dentro del proceso. En ambos casos se trata siempre de espacios lineales, paralelos y homogéneos en dimensión. En la versión publicada en la obra completa, la orientación de las bóvedas aparece de forma nítida en sentido norte-sur construyendo la casa por yuxtaposición de elementos estructurales. La direccionalidad de las bóvedas queda ligada así a las vistas y la ventilación ${ }^{13}$.
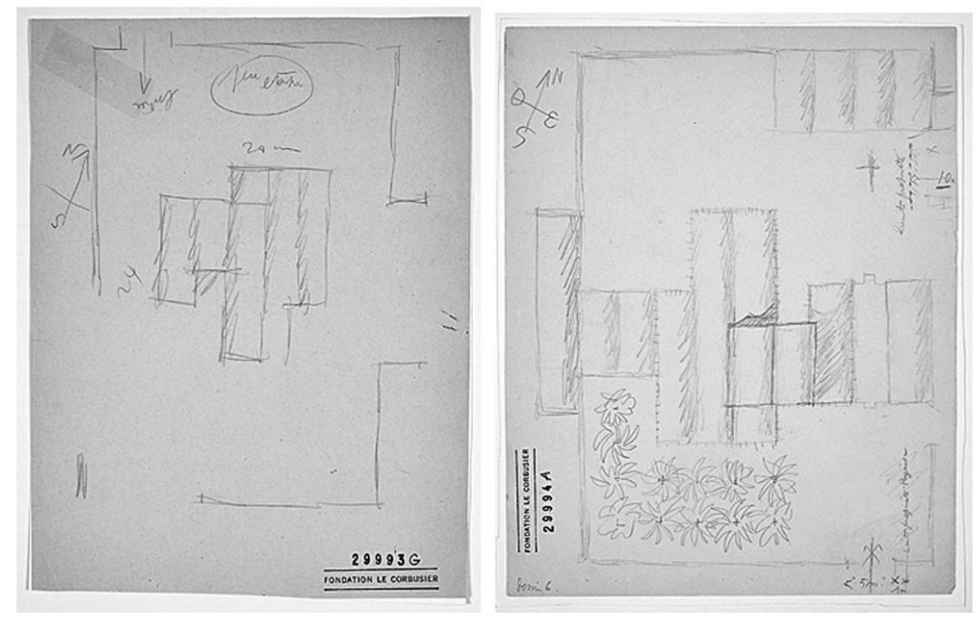

5. Dibujos originales FLC 29993G-CFLC-ADAGP y FLC 29994A-@FLC-ADAGP.

A pesar del carácter marcadamente longitudinal de todas las propuestas, se observa cómo dentro de ese orden paralelo aparece otro en sentido trasversal que define nuevos espacios. De este modo la casa puede entenderse como una sucesión de bandas, de nuevo paralelas, pero perpendiculares a la dirección de las bóvedas. En ellas encontramos dos categorías de espacios: por una parte aquellos definidos entre muros, como estancias acotadas (a veces equipadas alojando mobiliario); y por otro lado unos ámbitos continuos, diáfanos y pasantes que atraviesan toda la longitud de la casa conectando los espacios definidos bajo las bóvedas. Con ello se pone de manifiesto que el aparente carácter longitudinal de la casa es ficticio puesto que la organización de la misma responde a una trama regular en dos direcciones.

\footnotetext{
${ }^{10}$ FLC 29993B.

${ }^{11}$ FLC 29990D.

${ }^{12}$ Según se aprecia en la comparación entre las propuestas de cubierta en las axonometrías analíticas.

${ }^{13}$ FLC 29993 G y FLC 29994A.
} 
La definición constructiva de la estructura portante determina la materialización de los espacios. Se estandariza y homogeniza mediante tres tipos de muros que sujetan las bóvedas: pilar cuadrado, muro de medio módulo y muro completo, lo que da lugar a un juego versátil de combinaciones. Aunque es escasa la documentación existente en este aspecto si se hace referencia a que el módulo del proyecto queda en relación con la construcción de las bóvedas catalanas realizadas mediante ladrillo. Se produce una ambientación de los interiores mediante la combinación de tres materiales: la mampostería vista, las bóvedas encaladas de blanco y los cerramientos de madera. El suelo también queda planteado en madera. La independencia entre estructura y plementería como mecanismo arquitectónico aparece reflejado en este proceso de proyecto. De este modo, frente a la estructura portante masiva de muros y bóvedas, el cerramiento se proyecta mediante una carpintería en entramado asegurando la compartimentación y especialización de los huecos ${ }^{14}$. Éstos quedan materializados mediante paneles opacos, transparentes o translucidos, según las necesidades.

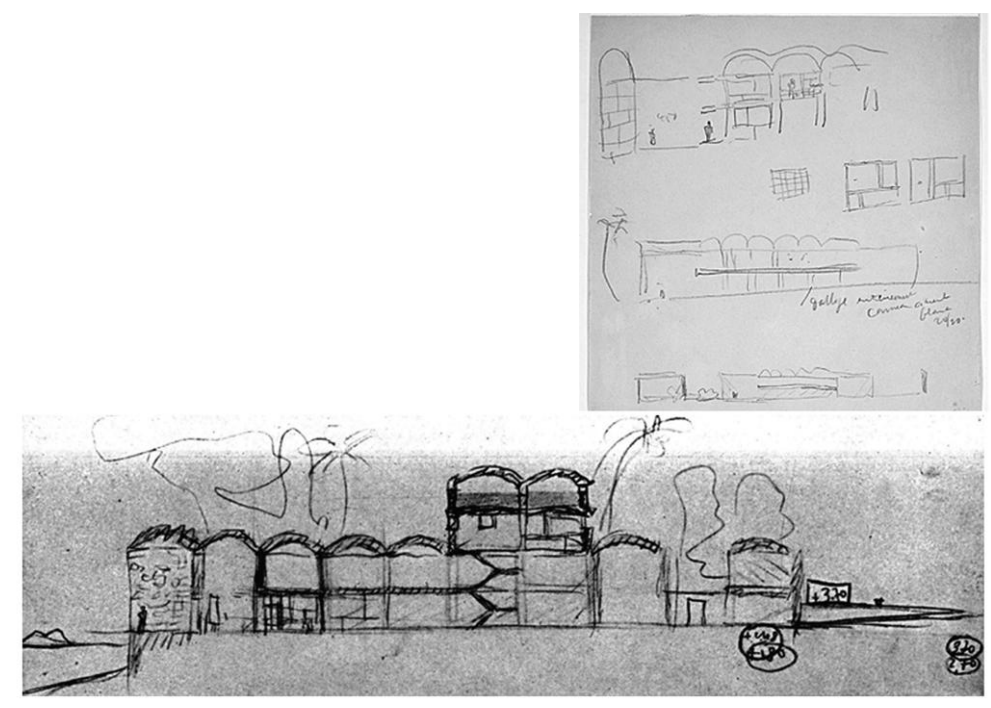

6. Fragmentos de los dibujos originales FLC 29993H-OFLC-ADAGP y FLC 29994D-@FLC-ADAGP de secciones en las que aparecen las alturas de los espacios.

La altura libre de los espacios es otro de los parámetros esenciales en la configuración de los mismos. Se maneja la utilización tanto de espacios de altura sencilla como de ámbitos de doble dimensión. De este modo la casa se convierte en una secuencia de espacios comprimidos y dilatados concatenados. La doble escala aparece en los extremos de la sección aumentando la presencia de la casa en relación con el paisaje mientras que los espacios interiores se configuran mediante una única altura ${ }^{15}$.

Respecto a la definición funcional de la casa, los dibujos aportan algunas anotaciones escritas sobre el posible uso de los espacios e incluso llega a dibujarse algún mobiliario que determina un programa doméstico configurado por partes ${ }^{16}$.

\footnotetext{
${ }^{14}$ FLC $29993 \mathrm{H}$.

${ }^{15}$ FLC $29993 \mathrm{H}$.

${ }^{16}$ FLC 29994B y FLC 29995A.
} 


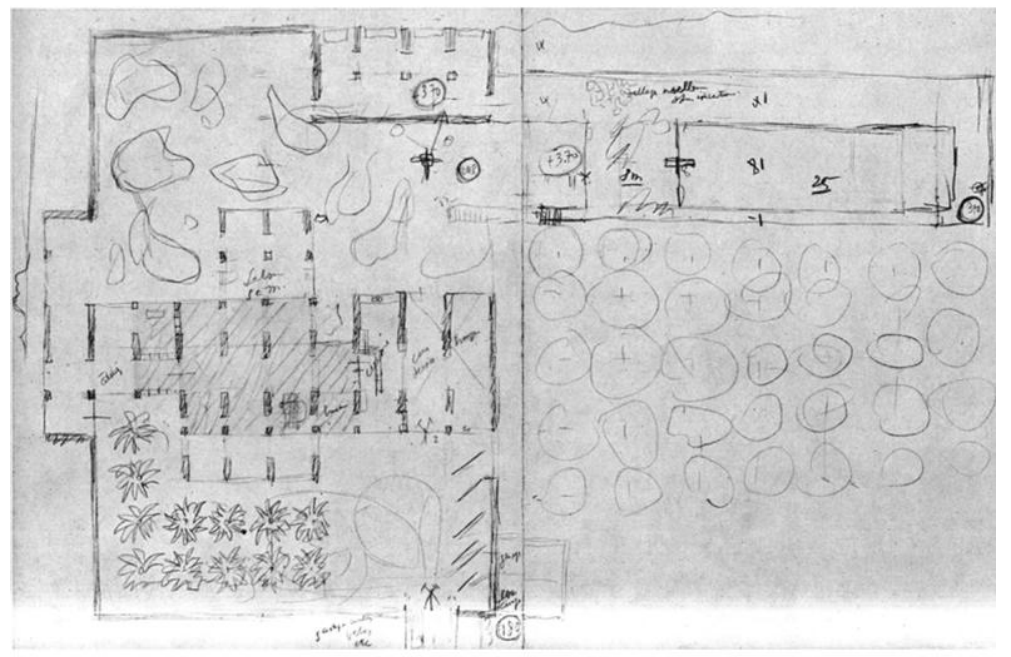

7. Fragmento del dibujo original FLC 29995A-CFLC-ADAGP de planta baja de la propuesta.

\subsection{Los miradores}

El proyecto muestra constantemente la contradicción entre una casa introvertida dentro de un recinto frente a un espacio extrovertido que vierte al paisaje exterior lejano. A partir de esta dualidad, la propuesta incorpora una reflexión permanente sobre la definición de espacios destinados a la apertura de la mirada al horizonte y las vistas, debido a la situación privilegiada de la casa sobre un acantilado. Se trabaja con dos direcciones y visiones principales: al norte hacia el mar y al oeste a la bahía de Cherchell y la montaña de Chenoua. Se produce la abstracción de las condiciones que le interesan del paisaje a través de la capacidad de síntesis sobre la conformación del mismo ${ }^{17}$. La mirada precisa y determinada queda asociada a los dibujos en planta ${ }^{18}$ y el paisaje aparece como recorte, enmarcado por la arquitectura.

Se proyectan estancias configuradas para mirar solamente hacia dos puntos concretos ${ }^{19}$. Aparece por vez primera la idea de conformación de espacios específicos contemplativos, destinados a observar el horizonte mediante zonas donde la tapia se dilata y adquiere espesor ${ }^{20}$. El proceso de proyecto sobre la configuración de estos espacios arroja una profunda reflexión que da lugar a distintas versiones dentro de un proceso secuencial en el que se define la organización de los mismos. Desde un primer momento, casi todos los esquemas dibujados separan los recortes en la tapia del recinto para poder mirar de forma independiente a las dos vistas definidas (norte y oeste) menos en el plano "FLC 29990D" donde la esquina del recinto relacionada con las vistas queda completamente abierta. Estos dos planos de visión separados no quedan vinculados a la casa en la mayoría de los croquis adquiriendo una formalización parecida en la serie de planos "FLC 29990A", "FLC 29990C" y "FLC 29992B". En estos casos siempre existe un muro ciego que separa los recortes de la tapia para la mirada.

\footnotetext{
${ }^{17}$ FLC 29990B.

${ }^{18}$ FLC 29990C.

${ }^{19}$ Así por ejemplo incluso hay algún dibujo, como es el caso del plano FLC 29992A donde la casa aparece desplazada el vértice superior izquierdo de la parcela con una posición totalmente polarizada hacia las vistas.

${ }^{20}$ FLC 29993A.
} 

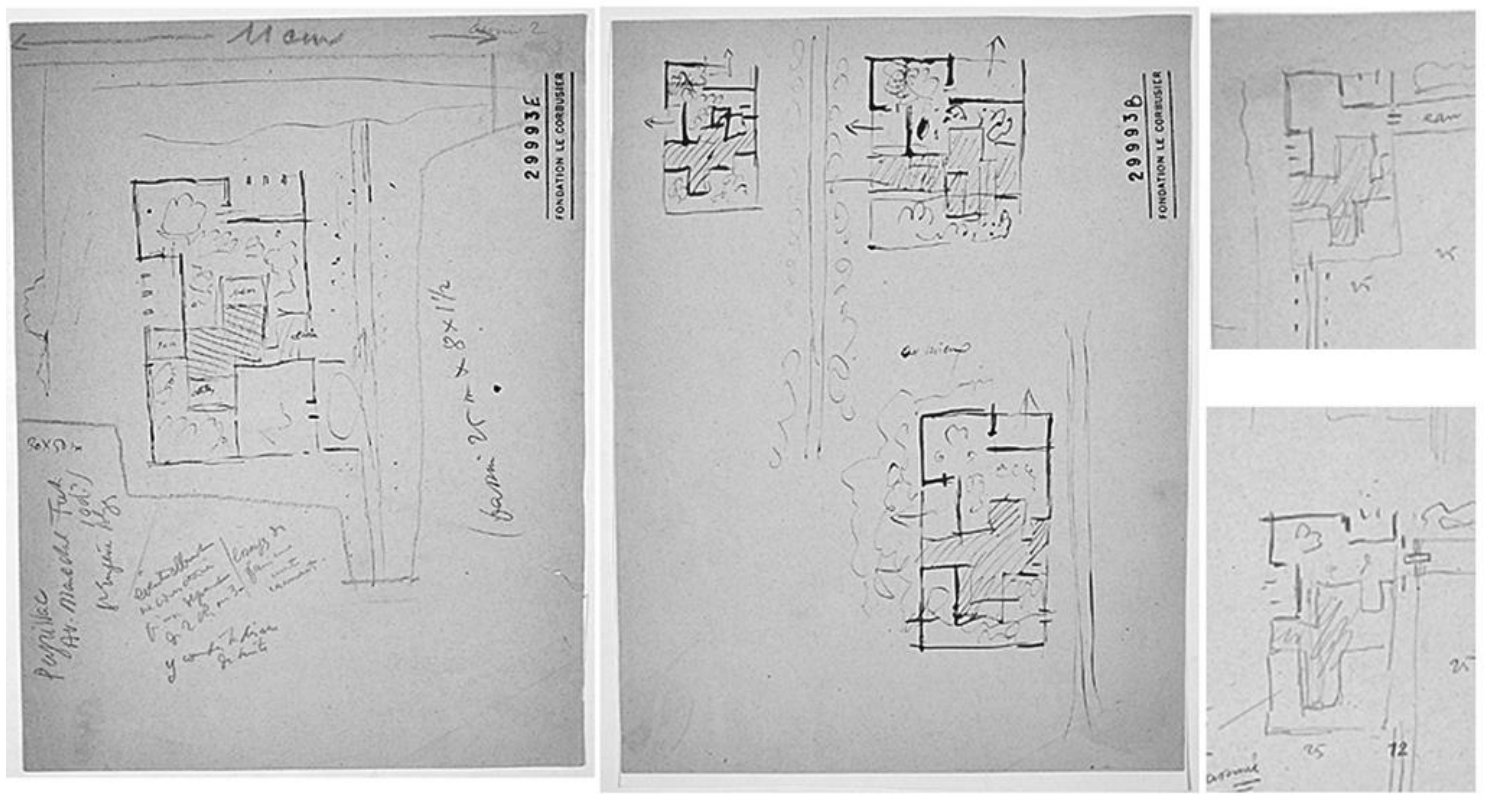

8. Dibujos originales FLC 29993E-CFLC-ADAGP, FLC 29993B-CFLC-ADAGP y fragmentos del dibujo FLC 29993CCFLC-ADAGP.

Éstos quedan ortogonales entre sí y orientados directamente al norte y al oeste. Una versión más avanzada se produce en el plano FLC 29993E cuando el diedro noroeste del recinto se plantea como elemento ciego de forma que la zona abierta a la bahía se desplaza de su posición anterior y se acerca a la casa. Una vez especializados los espacios para mirar, uno vinculado a la casa y otro independiente a ella, se produce un nuevo catálogo de soluciones donde todos ellos se sitúan todavía en el interior del recinto. En esta nueva serie ${ }^{21}$ se van definiendo y acotando los espacios a través de muros convirtiéndolos paulatinamente en espacios cada vez más cualificados y autónomos. Los muros se van plegando hasta encerrar las zonas abiertas al horizonte que aparecen como final perspectivo de la secuencia de recorridos a través de los espacios de la casa. Se realiza la construcción de entradas angostas y laterales a los miradores acentuando la sensación de descompresión espacial en la transición que va lo de introvertido de la vivienda a lo extrovertido de la mirada al paisaje lejano ${ }^{22}$. Un hito dentro del proceso del proyecto aparece cuando se produce la intersección entre la casa y los espacios mirador. De este modo es la propia casa la que se proyecta al exterior ${ }^{23}$. En la versión más desarrollada esta ligazón entre la vivienda y el mirador ocasiona que, como novedad, aparezca éste último en una posición definitivamente exterior al recinto. Así queda completada la reflexión sobre la configuración de los miradores.

\footnotetext{
${ }^{21}$ Representada por los dibujos del plano FLC 29993B.

${ }^{22}$ FLC 29993C.

${ }^{23}$ FLC 29993E.
} 

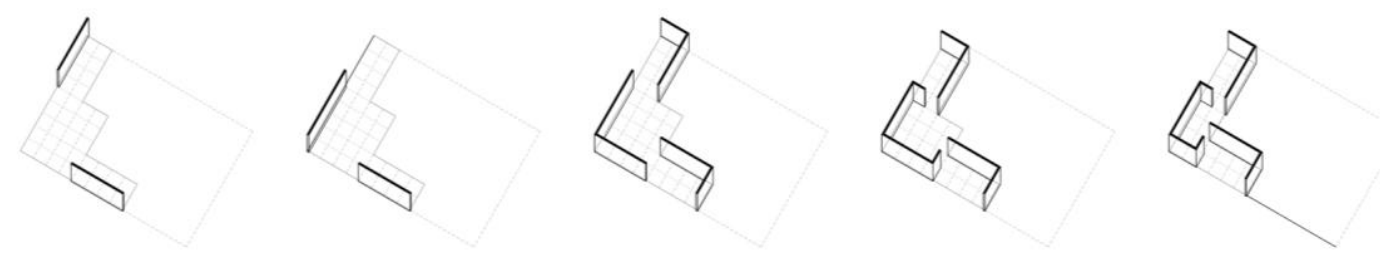

9. Versiones sobre la configuración del diedro noroeste del recinto con los espacios destinados a la mirada del paisaje.

\subsection{Los patios}

La construcción del interior del recinto como un sistema de llenos y vacíos determina una serie de espacios exteriores, a modo de patios, que adquieren autonomía tanto espacial como programática. Se emplea una estructura basada en la suma de elementos equivalentes a partir de la adición de módulos creando un sistema celular, a priori flexible, donde las partes pueden ser intercambiables sin que aparezca una forma definitiva ni predeterminada. Se produce una interacción entre las partes construidas y las exteriores ya que ambas pertenecen a una estructura jerárquicamente superior que las organiza: la trama regular y modulada que define su geometría.

La organización de los patios siempre queda vinculada y determinada por la posición fija que adquiere la casa dentro del recinto. En todos los dibujos la vivienda fracciona en dos partes diferenciadas a los vacíos. De este modo aparece un patio hacia el sur y otro hacia el norte. El primero de ellos sirve tanto de acceso como para la colocación de la vegetación mediante palmeras que tamicen el soleamiento y generen sombra arrojada sobre la casa; y el segundo de ellos, de carácter más artificial, organiza los accesos a los miradores. Así los patios quedan diferenciados en dos vacíos contrapuestos: un vacío artificial al norte y uno colmatado de vegetación natural en el $\operatorname{sur}^{24}$. Se crea la construcción de paisajes domésticos especializados en los patios, por una parte la sala hipóstila de palmeras, y por otra la multiplicidad de colinas artificiales. Estas diferencias aparecen por ejemplo en el plano "FLC 29990C" donde el color categoriza los patios y por tanto muestra las diferencias existentes entre ellos y la especialización de los usos asociados. Se realizan también estudios del orden de plantación de la vegetación asociado a la trama modulada ${ }^{25}$. Es interesante destacar cómo las distintas configuraciones en los acceso a los miradores genera la fragmentación de las diferentes zonas del patio norte. Así es cómo en los planos "FLC 29993B" y "FLC 29993C" aparece la reflexión sobre el uso específico del segmento de patio que queda determinado por el diedro ciego en el vértice noroeste del recinto. La colocación mas cercana de los muros de los dos miradores provocan que este espacio libre gane independencia espacial e incluso se grafíe de forma distinta al resto de ámbitos del patio norte.

\footnotetext{
${ }^{24}$ FLC 29994A.

${ }^{25}$ FLC 29993D.
} 


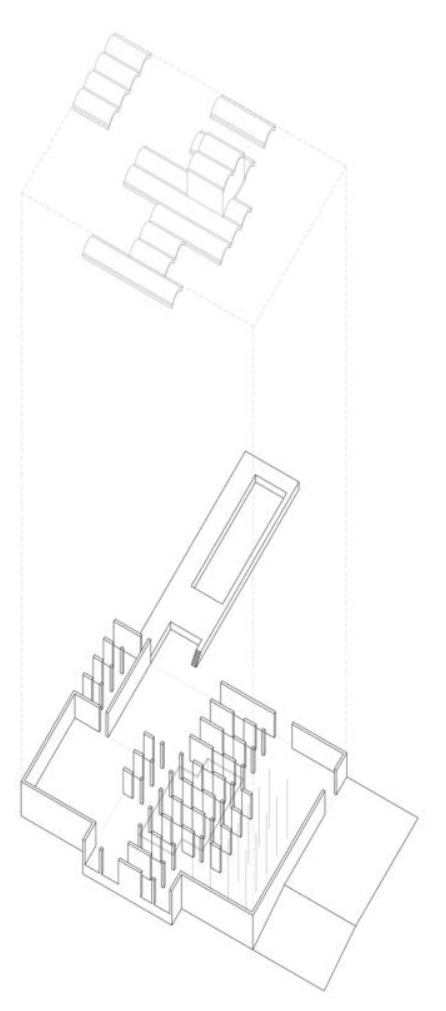

10. Axonometría de conjunto.

\section{Síntesis}

A modo de conclusiones de la investigación se realiza un apartado resumen en el que se hace referencia, de forma sintética, a los temas de proyecto tratados por Le Corbusier a lo largo del proceso.

\subsection{El espacio umbral}

La planta de la Residencia en Argel habla de dialéctica. Por un lado propone el lleno frente al vacío: el lleno de lo construido y el vacío de los patios. En los propios patios se produce un juego doble: el patio sur se conforma como una sala hipóstila llena de palmeras; el patio norte es un espacio topográfico que incluye una serie de pequeñas elevaciones de terreno configuradas como colinas artificiales. La organización de la posición de la casa dentro del recinto responde a un esquema muy similar al de la villa Savoye determinando un patio delantero orientado a sur para la iluminación y otro a norte para las vistas ${ }^{26}$. Cuando se excava un terreno para construir la cimentación se trasladan las tierras a la periferia de la ciudad. Le Corbusier reconoce la mala utilización de estas tierras y su valor por lo que decide aprovechar este material natural junto a lo edificado, generando nuevas topografías que ayudarán a integrar la pieza en su entorno y construir así nuevos paisajes artificiales asociados a los patios.

${ }^{26}$ FLC 29992 C. 


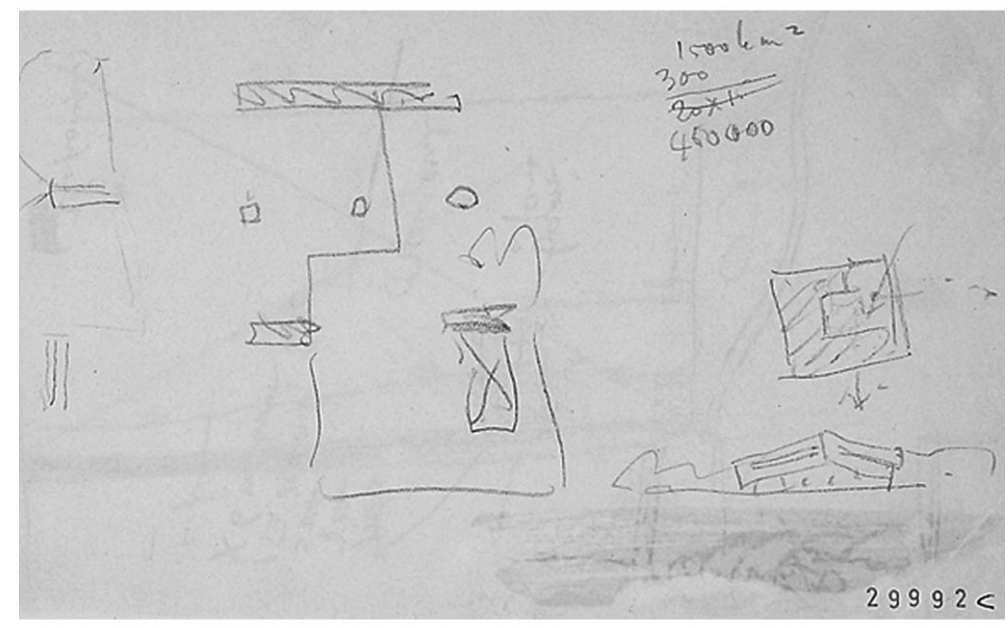

11. Dibujo original FLC 29992C-CFLC-ADAGP en el que se recoge un croquis de la villa Savoye.

Se plantean fachadas a cuatro orientaciones distintas y cuatro paisajes diferentes: al norte el mar; al oeste la montaña; al sur el acceso desde la carretera; al este la zona cultivada y la alberca que servirá para regar los cultivos de tomates. Lo natural frente a lo artificial. Y el sol frente a la sombra. La vivienda es un espacio umbral abierto en el que se busca la sombra y la ventilación cruzada entre la combinación de espacios abiertos, cerrados e intermedios exteriores pero cubiertos.

\subsection{El muro grueso y el ojo que mira}
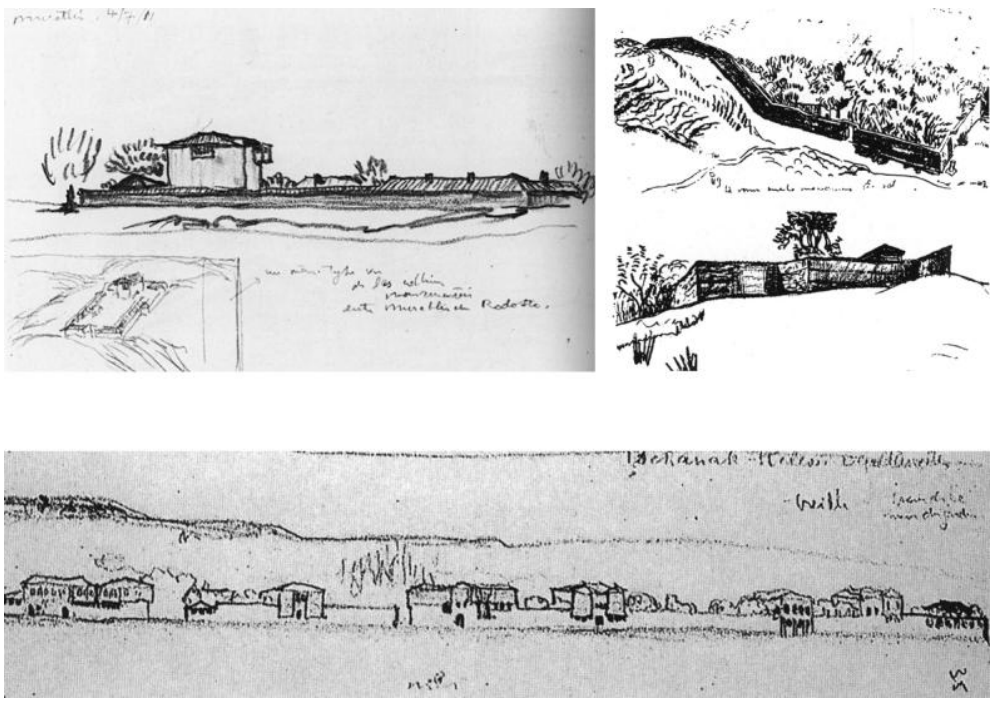

12. Dibujos originales de casas tradicionales de Turquía realizados por Le Corbusier durante su viaje a Oriente en 1911.

En las secciones del proyecto se descubre el grosor del recinto envolvente. ¿Qué significa un muro grueso? Por un lado, supone llevar al extremo la separación entre interior y exterior, señalar estos ámbitos como mundos distintos. Es también un gesto defensivo, la protección frente un entorno hostil ${ }^{27}$. Además, el grosor del muro

\footnotetext{
${ }^{27}$ El propio Le Corbusier señala en la memoria del proyecto publicado "Primera idea: un recinto cerrado con un perro malo..." Véase Le Corbusier: Oeuvre complète Volume 4 1938-46, 11th edition. Basel: Birkhäuser-Publishers for Architecture, 1999. p. 116.
} 
genera sombra. Y lo más importante para Le Corbusier: un muro grueso posibilita el enmarcado, pues la naturaleza es más interesante para el maestro a través de la arquitectura ${ }^{28}$. En los croquis realizados, Le Corbusier da una especial importancia al paisaje circundante: el mar y el horizonte son protagonistas a través de los huecos que se abren al exterior ${ }^{29}$. Para conseguir las visiones lejanas, Le Corbusier vincula la construcción al recinto y abre un único hueco de gran tamaño, ${ }^{30}$ semejante al ojo del cíclope. El ojo que mira es un eco del gran hueco que domina el paisaje urbano en las propuestas de los años 20 para los Inmuebles Villa. Y es también la transposición a la arquitectura moderna de la idea subyacente en los miradores de Estambul dibujados por Le Corbusier en su viaje a Oriente: un recinto que en determinados puntos se hace más grueso y eleva para formar el mirador ${ }^{31}$.

\subsection{El espacio diagonal}

La casa se estructura mediante muros de carga paralelos y bóvedas catalanas apoyadas en éstos. Este sistema, es utilizado por Le Corbusier por primera vez en el Chalet Poiret de 1916 y en el proyecto de las Casas Monol de 1919 y es una constante en otros proyectos. En proyectos posteriores al desarrollo del objeto de estudio de esta investigación que data del año 1942, Le Corbusier también emplea este sistema en el proyecto para SainteBaume de 1948, en las Casas Jaoul en París de 1952 o la Casa Sarabhai en Ahmedabad de 1955) ${ }^{32}$. Existe también una clara vinculación entre este tipo de espacios generados bajo bóvedas de cañón paralelas y los que aparecen en la Porticus Aemilia de Roma ${ }^{33}$. La aparición de las bóvedas sobre el muro perimetral conforma un nuevo skyline en el proyecto. Los muros de carga permiten la división de los planos estructurales en partes más pequeñas y gracias a ello, la aparición de diagonales visuales en planta a través de una trama ortogonal pero fragmentada. Se rompe el concepto de espacio lineal apareciendo una nueva complejidad vinculada con la diagonal.

\subsection{Patio y pabellón}

Le Corbusier propone, en el proyecto de Argel aquí estudiado, un recinto en cuyo interior se dispondrá la residencia. Toda la combinación arquitectónica queda basada sobre el principio que conduce a un juego sutil de llenos y vacíos en relación con las formas fundamentales de la tradición mediterránea. Casa y envolvente forman una simbiosis y los muros de carga paralelos que estructuran la vivienda se ligan, en determinados puntos, al muro perimetral que conforma el recinto. La vivienda es pabellón y casa patio a la vez. Es pabellón entre el bosque de palmeras y la topografía artificial.

\footnotetext{
${ }^{28}$ En el proyecto del Ministerio de Educación en Río de Janeiro (1936-1945) Le Corbusier explica gráficamente el valor de la naturaleza enmarcada por la arquitectura. Véase Le Corbusier: Oeuvre complète Volume 4 1938-46, 11th edition. Basel: Birkhäuser-Publishers for Architecture, 1999. pp. 80-81.

${ }^{29}$ En la publicación de este proyecto en la obra completa se definen los elementos arquitectónicos: el horizonte, el mar, la terraza, los pilares de obra, las bóvedas. Véase Le Corbusier: Oeuvre complète Volume 4 1938-46, 11th edition. Basel: Birkhäuser-Publishers for Architecture, 1999. p. 119.

${ }^{30} \mathrm{El}$ hueco que se abre al paisaje se dispone en zonas de doble altura.

${ }^{31}$ Véase Gresleri, Giuliano: Le Corbusier Viaggio in Oriente, Venecia: Marsilio Editori, 1984. pp. 223, 246-247.

${ }^{32}$ La semejanza con las Casas Jaoul y la Casa Sarabhai se extiende también a la inserción de paneles de madera en los alzados cortos.

${ }^{33}$ Tal y como destaca S.von Moos en su libro "Le Corbusier. Elements of a Synthesis".
} 


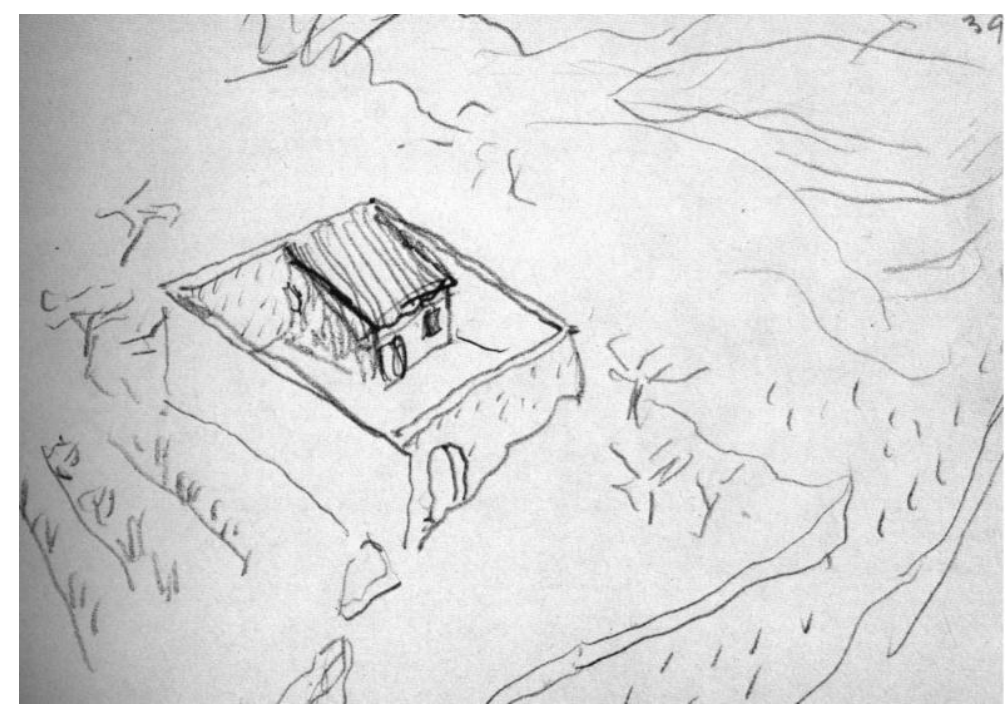

13. Dibujo de Le Corbusier sobre una casa con patio tradicional.

Esto conduce a una doble lectura de la casa a través de su condición introvertida y extrovertida simultáneamente. La construcción como lugar acotado y protegido aparece ligada a la secuencia compleja de espacios interiores, intermedios y exteriores. Estos sistemas se implementan con la versión de la casa como mirador. Al igual que sucedía en los croquis iniciales de la "Maison de week-end" en La Celle-Saint-Cloud de 1934, se realizan estancias en plantas altas que se elevan sobre las tapias, permitiendo que la mirada escape del recinto y se dirija hacia el horizonte.

\section{Procedencia de las imágenes}

(1). Dibujo realizado por los autores de la comunicación: arrroyopemjean+sanz@

(2). Brooks, H. Allen: The Le Corbusier Archive. New York-London: Garland Publishing, Inc., Fondation Le Corbusier, 1982-1984.

(3). Brooks, H. Allen: The Le Corbusier Archive. New York-London: Garland Publishing, Inc., Fondation Le Corbusier, 1982-1984.

(4). Dibujo realizado por los autores de la comunicación: arrroyopemjean+sanz@

(5). Brooks, H. Allen: The Le Corbusier Archive. New York-London: Garland Publishing, Inc., Fondation Le Corbusier, 1982-1984.

(6). Brooks, H. Allen: The Le Corbusier Archive. New York-London: Garland Publishing, Inc., Fondation Le Corbusier, 1982-1984.

(7). Brooks, H. Allen: The Le Corbusier Archive. New York-London: Garland Publishing, Inc., Fondation Le Corbusier, 1982-1984.

(8). Brooks, H. Allen: The Le Corbusier Archive. New York-London: Garland Publishing, Inc., Fondation Le Corbusier, 1982-1984.

(9). Dibujo realizado por los autores de la comunicación: arrroyopemjean+sanz@

(10). Dibujo realizado por los autores de la comunicación: arrroyopemjean+sanz@

(11). Brooks, H. Allen: The Le Corbusier Archive. New York-London: Garland Publishing, Inc., Fondation Le Corbusier, 1982-1984.

(12). Gresleri, Giuliano: Le Corbusier Viaggio in Oriente. Venecia: Marsilio Editori, 1984.

(13) Le Corbusier: Sketchbooks Volume 1, 1914-1948. London: Thames and Hudson, 1981. 


\section{Bibliografía}

Boesiger, Willy: Le Corbusier. Barcelona: Editorial Gustavo Gili, 1985.

Brooks, H. Allen: The Le Corbusier Archive. New York-London: Garland Publishing, Inc., Fondation Le Corbusier, 1982-1984.

Gresleri, Giuliano: Le Corbusier Viaggio in Oriente. Venecia: Marsilio Editori, 1984.

Le Corbusier: Oeuvre complète Volume 4 1938-46, $11^{\text {th }}$ ed. Basel: Birkhäuser-Publishers for Architecture, 1999.

Le Corbusier: Precisiones respecto a un estado actual de la arquitectura y el urbanismo. Barcelona: Ediciones Apóstrofe, 1999.

Le Corbusier: Sketchbooks Volume 1, 1914-1948. London: Thames and Hudson, 1981.

Moos, Stanislaus von: Le Corbusier: elements of a synthesis. Cambridge, Masasachusetts: The Mit Press, 1979.

Regidor, Mabel: "Visita a una casa de Alejandro de la Sota". En Periferia. Junio 1991, N.10. pp. 93-95.

Vidotto, Marco: Alison + Peter Smithson, Obras y Proyectos. Barcelona: Editorial Gustavo Gili, 1997. 